\title{
Anisotropy, Size, and Aspect Ratio Effects on Micropillar Compression of Al-SiC Nanolaminate Composites
}

\author{
C. Mayer ${ }^{1}$, L.W. Yang ${ }^{2}$, S.S. Singh ${ }^{1}$, J. Llorca ${ }^{2,3}$, J.M. Molina-Aldareguia ${ }^{2}$, Y.L. Shen ${ }^{4}$, N. \\ Chawla $^{1}$ * \\ ${ }^{1}$ Materials Science and Engineering, Arizona State University, Tempe, AZ 85287-6106 \\ ${ }^{2}$ IMDEA Materials Institute, c/ Eric Kandel 2, 28906 Getafe, Madrid, Spain \\ ${ }^{3}$ Department of Materials Science. Polytechnic University of Madrid. E. T. S. de Ingenieros de \\ Caminos, 28040 - Madrid, Spain \\ ${ }^{4}$ Department of Mechanical Engineering, University of New Mexico, Albuquerque, NM, USA
}

Submitted to:

\section{Acta Materialia}

February 2016

*Corresponding author: nchawla@ asu.edu, 480-965-2402,

501 E. Tyler Mall, ECG 303

Arizona State University

Tempe, AZ 85287-6106 


\begin{abstract}
Metal-ceramic nanolaminate composites show promise as high strength and toughness materials. Micropillar compression was used to characterize the mechanical behavior of Al-SiC multilayers in different orientations including loading at $0^{\circ}, 45^{\circ}$ and $90^{\circ}$ with respect to the direction of the layers. The $0^{\circ}$ orientation showed the highest strength while the $45^{\circ}$ orientation showed the lowest strength. Each orientation showed unique deformation behavior. Effects of pillar size and aspect ratio were also studied. Higher compressive strengths were observed in smaller pillars for all orientations. This effect was shown to be due to a lower probability of flaws using Weibull statistics. Additionally, changes in the aspect ratio was shown to have no significant effect on the behavior except an increase in the strain to failure in the $0^{\circ}$ orientation. Finite element analysis (FEA) was used to simulate and understand the effect of these parameters on the deformation behavior.
\end{abstract}

Keywords: multilayer, nanocomposite, orientation dependence, finite elements, Weibull analysis 


\section{List of Figures}

Figure 1: Schematic of the pillar orientations tested and SEM images of $2 \times 4 \mu \mathrm{m}$ pillars prior to testing.

Figure 2: Experimental engineering stress strain curves showing the effect of layer orientation on mechanical response for different geometries.

Figure 3: SEM images of $1 \times 2 \mu \mathrm{m}$ pillars and cross sections following compression for $0^{\circ}$ (a and b), $90^{\circ}$ (c and d), and $45^{\circ}$ (e and f) orientations. The deformation behavior shows large differences with the $0^{\circ}$ and $45^{\circ}$ orientations being strongly influenced by the buckling of the layers. The $90^{\circ}$ orientation shows extrusion of the $\mathrm{Al}$ layers.

Figure 4: FEA stress contours at $6 \%$ strain showing the effect of $0 \mathrm{~nm}(\mathrm{a}, \mathrm{c}$, and e) and $45 \mathrm{~nm}(\mathrm{~b}, \mathrm{~d}$, and f) amplitude waviness on the deformation behavior for all three orientations. Due to lack of convergence e) is only able to be modeled to $2.5 \%$ strain (also note the difference in contour levels).

Figure 5: Comparison of engineering stress strain curves for a representative $2 \times 4 \mu \mathrm{m}$ pillar to FEA simulations (also $2 \mathrm{x} 4 \mu \mathrm{m}$ ) having waviness amplitudes of 0,15 and $45 \mathrm{~nm}$ in each orientation.

Figure 6: Experimental engineering stress-strain curves showing the effect of pillar size and aspect ratio on mechanical behavior.

Figure 7: Weibull plot of the fracture stresses of $1 \times 2$ and $2 \times 4 \mu \mathrm{m}$ pillars (in the $90^{\circ}$ orientation). The fact that both sets of data fall on a single linear fit line indicates that the increase in mean strength of the $1 \times 2$ $\mu \mathrm{m}$ pillars can be attributed to lower probability of a strength limiting flaw and is not due to FIB damage or a true material size effect.

Figure 8: Simulated deformation behavior of $2 \times 6$ and $2 \times 4 \mu \mathrm{m}$ pillars (same contour scale as Figure 4) showing that the height of the instability is independent of the overall pillar height. This leads to a decreased apparent strain to failure, as shown in the engineering stress-strain curve. 


\section{Introduction}

Laminate composite materials have long been used to obtain material properties which are a combination of the properties of their constituents. Reduction of the dimensions of the individual constituents of these laminates to the nanoscale $(\leq 100 \mathrm{~nm}$ layer thickness) has allowed these materials to exhibit unique properties which are superior to that of their macroscale counterparts. These nanolaminate composites show promise in applications requiring properties such as high strength, toughness, and wear resistance $[1,2,3,4,5,6]$, as well as biocompatibility [7], and certain optical properties [8]. Additionally, like macro-scale composites, the properties of these materials can be tailored by varying composition and layer thicknesses to obtain optimum properties.

Metal-ceramic nanolaminates were chosen as the material of interest in this study. From a practical perspective, these materials exhibit a combination of high toughness and strength which is attractive for many applications $[9,10]$. Scientifically, these materials provide an ideal environment to study plasticity under extreme degrees of constraint due to the large elastic and strength mismatch between the layers. However due to the limited volume of material which is able to be deposited, the mechanical characterization methods available are limited to micro scale techniques.

The orientation dependence of macro-scale laminated composites has been studied previously, with the majority of the studies focusing on the orientations implications on fracture toughness $[11,12,13]$. Research performed by Roy et al. [12] is very analogous to the present study, where the compressive behavior of bulk aluminum - aluminum oxide laminar composites (20-220 $\mu \mathrm{m}$ individual lamina thickness) was determined as a function of laminate orientation. 
This work showed that due to the varying load transfer to the reinforcement, as the angle between the lamina and the loading axis increases there is a steep decrease in strength from $0^{\circ}$ to $30^{\circ}$, a minimum at $45^{\circ}$, and finally a slight increase at $90^{\circ}$. In addition, the strain the material is able to accommodate varies in the opposite manner, increasing as the load becomes more misaligned with the reinforcement phase.

Pillar compression is becoming an increasingly popular microscale mechanical testing technique $[6,14,15,16,17,18,19]$. This technique utilizes a flat punch along with the high load and displacement resolution of instrumented indentation in order to carry out extremely sensitive compression experiments. Pillar compression provides an approximately uniaxial and uniform stress state which also allows the stress strain behavior to be determined. The uniaxial stress state is particularly critical in characterizing the anisotropy, as shown by our previous attempts to characterize this dependence using nanoindentation [20]. The results of the experimental Berkovich indentations and finite element modeling from that study showed that, due to the complex stress state caused by the indenter geometry, the modulus determined through indentation shows a much weaker dependence on orientation than what is predicted through the classical laminate theory. Spherical nanoindentation has also been used to determine the stress strain response and elastic anisotropy in other materials [21], however micropillar compression has the advantage of a clearly defined stress state which is more appropriate for this study.

In order to determine the anisotropy in the deformation behavior of these materials, three orientations, with the loading axis forming $0^{\circ}, 90^{\circ}$, and $45^{\circ}$ with respect to the layer direction, were characterized using pillar compression. Pillars compressed perpendicular to the nanolaminate surface $\left(90^{\circ}\right)$ subject the layers to a nearly isostress condition, while pillars compressed in the transverse direction $\left(0^{\circ}\right)$ load the layers in a nearly isostrain condition. 
Finally, pillars oriented at $45^{\circ}$ with respect to the laminate surface generate the largest amount of shear stresses parallel to the layers.

The size effect phenomenon in pillar compression, where the flow stress of the materials increases as the size of the pillars is reduced, has been documented in a number of cases in single phase materials at small scale lengths $[14,15,17,22]$. Having microstructural features smaller than the pillar size precludes one of the most common mechanism the size effect is attributed to, namely dislocation starvation. Therefore, this effect requires further investigation in nanostructured materials where the testing geometry is still much larger than the structural features.

This work aims to characterize two aspects of nanolaminate behavior which have not been addressed previously. First, as nearly all previous experimental efforts have been focused only on loading normal to the layers, there is a need to characterize the properties of these composites in other orientations to determine if the classical laminate behavior is still applicable at the nanometer length scale. Second, it is necessary to determine both if and why geometric factors, namely the size and aspect ratio of these pillars, have an effect on the measured response. In order to achieve these aims, the quantitative measurement of the material response gained through pillar compression will be paired with both post deformation imaging and finite element modeling in order to provide a comprehensive understanding of the deformation mechanisms dictating the behavior.

\section{Materials and Experimental Procedure}


The metal-ceramic nanolaminate composites were fabricated using an automated magnetron sputtering procedure, the details of which are given elsewhere [23, 24]. The samples used in this study consisted of alternating layers of $\mathrm{Al}$ and $\mathrm{SiC}$, each having an individual layer thickness of approximately $50 \mathrm{~nm}$. These layers were deposited sequentially until a total multilayer thickness of approximately $15 \mu \mathrm{m}$ was obtained ( $\sim 300$ individual layers). The $0^{\circ}$ sample was able to be used for pillar compression without further preparation. The $90^{\circ}$ and $45^{\circ}$ samples, however, required mounting in epoxy and polishing in order to expose the edge to be tested, shown schematically in Figure 1.

Pillar fabrication, post-mortem imaging and cross-sectioning were performed using a dual beam FIB operated at $30 \mathrm{keV}$ ion beam accelerating voltage, which provides a high milling rate, and $5 \mathrm{keV}$ electron beam accelerating voltage, which provides high imaging resolution. Pillar fabrication was performed using an annular milling procedure, which enables a higher throughput of pillars compared to lathe milling [6], but results in approximately $2^{\circ}$ of taper, as shown in Figure 1. For each orientation, pillars were milled with nominal dimensions of $2 \times 4$ $\mu \mathrm{m}, 2 \times 6 \mu \mathrm{m}$, and $1 \times 2 \mu \mathrm{m}$ (diameter by height) with a $20 \mu \mathrm{m}$ surrounding trench to allow clearance for the indenter. These pillar sizes were chosen in order to study the size effect as well as the effect of aspect ratio. Various ion beam currents were used depending on the material removal rate and precision needed, but final polishing of the surfaces was always carried out using ion currents below $50 \mathrm{pA}$ (at $30 \mathrm{keV}$ ).

Pillar compression was carried out using a commercial nanoindenter (Nanoindenter XPII, Agilent) equipped with a diamond flat punch. Samples were mounted to aluminum stubs for testing using a mounting adhesive. Tests were performed using constant displacement rates of 5 $\mathrm{nm} / \mathrm{s}, 10 \mathrm{~nm} / \mathrm{s}$, and $15 \mathrm{~nm} / \mathrm{s}$ for $1 \times 2 \mu \mathrm{m}, 2 \times 4 \mu \mathrm{m}$, and $2 \times 6 \mu \mathrm{m}$ pillars, respectively, yielding 
an initial strain rate of $2.5 \pm 0.2 \times 10^{-3} \mathrm{~s}^{-1}$ (mean \pm standard deviation) for all experiments. The drift rate for all tests was held below $0.05 \mathrm{~nm} / \mathrm{s}$. The stress and strain was calculated based off of the initial pillar dimensions, yielding engineering stress and strain.

The deformation of the micropillars was simulated by finite element modeling (FEM) using the commercial software Abaqus (Abaqus, v. 6.12, Dassault Systems Simulia Corp., Providence, R.I.). The simulations were performed in 2D plane strain conditions. The models consisted of a rigid flat punch, micropillars with layers oriented at $0^{\circ}, 45^{\circ}$, and $90^{\circ}$ with respect to the micropillar axis and base material. Three different pillar sizes were modeled to account for size and aspect ratio effects, with similar dimensions to the pillars tested experimentally. To match more precisely with experimental conditions, a $2^{\circ}$ taper was also included in the pillar models. In each case, the effect of layer waviness was accounted for by comparing the results of the simulations performed with micropillars containing flat layers, to those containing undulated layers. The undulated layers were modeled by imposing a standard sinusoidal waveform with a wavelength of $0.5 \mu \mathrm{m}$ and amplitudes of 15 and $45 \mathrm{~nm}$. The $45 \mathrm{~nm}$ amplitude is close to the amplitude observed experimentally, although there is considerable variability in the actual microstructure. All the pillar models were meshed by 4-node bilinear plane strain quadrilateral meshes (CPE4) with approximately 30,000 elements (after performing a mesh convergence study). Constraint boundary conditions were imposed at the bottom of the base material, while the rest of the surfaces were set free.

For all of the models, the $\mathrm{Al}$ and $\mathrm{SiC}$ layers were modeled as elastic perfectly plastic materials, with no strain hardening, due to the small layer thickness, which precludes any dislocation storage. The Young's modulus of $\mathrm{Al}$ and $\mathrm{SiC}$ were taken as $70 \mathrm{GPa}$ and $300 \mathrm{GPa}$, and the corresponding Poisson's ratios were 0.34 and 0.14 , respectively. The yield stress of Al 
was $935 \mathrm{MPa}$ [25], and the apparent yield stress of $\mathrm{SiC}$ was chosen as $7 \mathrm{GPa}$ (a large value estimated from nanoindentation results of $1 \mu \mathrm{m}$ thick monolithic SiC films). The base material was modeled as a purely elastic material, with the elastic modulus and Poisson's ratio estimated as an average value of the isostrain and isostress composite moduli between $\mathrm{Al}$ and $\mathrm{SiC}$. The Al$\mathrm{SiC}$ interfaces were considered perfectly bonded in all cases.

\section{Results and Discussion}

\subsection{Effect of layer orientation on deformation morphology}

Engineering stress-strain curves obtained from the pillar compression tests for different orientations are shown in Figure 2, where each plot, corresponds to a different pillar geometry, i.e., $1 \times 2 \mu \mathrm{m}, 2 \times 4 \mu \mathrm{m}$, and $2 \times 6 \mu \mathrm{m}$ respectively. The arrows indicate the strain-to-failure of the pillars.

Although there is an effect of pillar size (discussed in later sections), Figure 2 indicates that the same qualitative trends in strength with respect to orientation are observed for all 3 pillar sizes, with the $0^{\circ}$ orientation being the strongest, the $45^{\circ}$ orientation being the weakest, and the $90^{\circ}$ orientation having an intermediate strength. SEM images of the pillars after deformation are shown in Figure 3. For the $0^{\circ}$ orientation, Figure 3(a) shows that the strain is localized at the top of the pillar. This localization was due to the hard SiC layers buckling under the applied load, as shown in Figure 3(b), triggering the formation of kink bands and the complete collapse of the pillars. The formation of kink bands under parallel loading is not surprising considering the large strength difference between the $\mathrm{Al}$ and the $\mathrm{SiC}$ layers. This kink band formation has also been observed in bulk $\mathrm{Cu}-\mathrm{Nb}$ nanolaminates [26]. In the $\mathrm{Cu}-\mathrm{Nb}$ case, no cracking was observed in the metallic multilayers, and no pronounced load drop occurred as the kink bands developed. This is 
in contrast to the present study where discontinuities in the SiC layers can be seen at the boundary of the kink band (Figure 3(b)). Additionally there is a peak in the load, although since the indentation actuation is inherently load controlled due to the electrostatic actuator, this peak results in catastrophic failure and the magnitude of the stress drop is unknown. Interestingly, bending of the layers tended to localize along columnar boundaries (i.e. the troughs in the waviness where the radius of curvature is small) within the nanolaminate microstructure. These boundaries occur in deposition processes due to the shading from surrounding peaks in the waviness that is exaggerated as more layers are applied [27]. This shading results in lower densities and porosity at these boundaries [27], and combined with the small radius of curvature which acts as a stress concentration, leads to a weak point in the structure. For the $90^{\circ}$ orientation, fracture occurred in a more brittle fashion, leading to a mushroom type deformation of the pillars, as shown in Figure 3(c). As observed in Figure 3(d), this deformation pattern occurs due to the formation of vertical cracks on the SiC layers, triggered by the radial tensile stresses that develop in them with the plastic deformation of the Al layers [28]. The plastic deformation of the $\mathrm{Al}$ layers, constrained by the SiC layers, is evident by the small extrusions that develop at the free surface, as can be seen in the lower, less strained part of the pillar (Figure 3(c)). However, for larger strains at the top of the pillar, the SiC layers crack, and the Al layers plastically flow within the cracks, leading to the formation of a mushroom type morphology. The deformation behavior of the $45^{\circ}$ pillars in Figure 3(e) was also dominated by the buckling of the $\mathrm{SiC}$ layers. As seen in cross-section image (Figure 3(f)), shear occurred predominantly in the direction normal to the layers under the action of the applied stress, preferentially along the weaker columnar boundaries, as indicated by the white arrows, instead of along the Al-SiC interfaces. The lack of delamination at the Al-SiC interface is indicative that the interface is 
stronger than the Al layers under shear loading. The strength of this interface under loading conditions closer to pure shear has been demonstrated elsewhere [24]. As a result of the shear localization along the pre-existing columnar boundaries and the plastic deformation along the Al layers, the layers tended to rotate with strain to become perpendicular to the applied stress. As a result, the layers at the top of the pillar form an approximately $60^{\circ}$ angle with respect to the loading axis, as opposed to the average angle of $45^{\circ}$ that can be measured on the undeformed section of the pillar. As opposed to the $0^{\circ}$ and $90^{\circ}$ orientations, which fail at strains of around 0.05-0.07, depending on pillar geometry, this type of deformation allows the pillar to accommodate large amounts of strain without collapsing, as seen in both the stress-strain curves of Figure 2 and the images of the deformed pillars.

\subsection{Effect of layer waviness on deformation morphology}

Overall, the results confirmed, as demonstrated before [23], that the nanolaminates deformed by the constrained plastic deformation of the Al layers and that the Al-SiC interface was stronger than the Al layers in shear. However, the results also showed that fracture of the SiC layers, especially along pre-existing columnar boundaries, was the main mechanism responsible for the final failure. Moreover, the failure pattern was very sensitive to the loading direction, with micropillars oriented at $0^{\circ}$ and $45^{\circ}$ mainly collapsing by the buckling of the SiC layers, and the micropillars oriented at $90^{\circ}$ failing by their transverse cracking. It is well known that buckling is very sensitive to vertical alignment, so it was expected that the layer waviness present in the microstructure should have a strong influence on the failure strain.

In order to assess the effect of layer waviness on deformation morphology, the results of FEA simulations utilizing micropillars with undulated and flat layers are compared in Figure 4 
for the different loading directions. The figures on the left correspond to the von Mises stress contours micropillars with flat layers, while the figures on the right are those with undulated layers for a waviness amplitude of $45 \mathrm{~nm}$. The simulated stress-strain curves for flat and undulated layers are also compared in Figure 5, where the experimental results of a $2 \times 4$ pillar is also plotted for reference. Comparing the results for the pillars loaded at $0^{\circ}$, it is evident that the layer buckling at the top of the pillar encountered experimentally was only reproduced by the simulations considering undulated layers in Figure 4(b). Moreover, the stress-strain curve of the simulation with undulated layers predicted a maximum in the stress in Figure 5(a) (denoted by arrows), as a consequence of the buckling of the layers, as experimentally observed. Analogously, the simulations of pillars loaded at $45^{\circ}$ also showed a very different behavior for flat and undulated layers. For flat layers, shear predominantly occurred parallel to the layers, resulting in little strengthening contribution from the $\mathrm{SiC}$ layers because the $\mathrm{Al}$ layers provide an uninterrupted shear plane within the pillar. This is in contrast to the wavy structure where the undulation of the layers interrupts the shear plane, leading to the formation of shear bands perpendicular to the layers and domains where the layers are substantially rotated towards the applied stress. Moreover, while the stress-strain curve for flat layers of Figure 5(c) showed very little strain hardening, in agreement with the strain localization observed along some of the $\mathrm{Al}$ layers in Figure 4(e), the stress-strain curve for undulated layers displayed an increase in apparent strain hardening rate, in agreement with the experimental observations. Finally, in the case of the $90^{\circ}$ loading direction, the results of the simulations with flat (Figure 4(c)) and undulated (Figure 4(d)) layers were very similar, as well as the predicted stress-strain curves (Figure 5(b)), indicating a less significant effect of the layer waviness when loading perpendicular to the layers. 


\subsection{Pillar size effects}

Figure 6 summarizes the effect of pillar size and aspect ratio on the stress-strain curves as a function of layer orientation. A non-negligible size effect could be observed for all three orientations, with the $1 \times 2 \mu \mathrm{m}$ pillars having significantly higher fracture strengths than the $2 \mathrm{x}$ $4 \mu \mathrm{m}$ pillars. Size effects have been observed in micropillar testing of other materials, especially when testing single crystals of pure metals [17, 19, 29]. Dislocation starvation and the lack of dislocation sources have been proposed as the cause of this strength increase in other works [16, 18]. This mechanism is not likely the cause of the strengthening in the case of nanolaminates. While dislocations slip more or less unimpeded and leave the surface in the case of single-crystal 
micropillars, the interfaces represent strong barriers for dislocation transmission [30], forcing them to glide confined within single layers [31]. As shown by previous studies by Sun et al. [32], this type of deformation results in very little residual dislocation density. Considering that the layer thickness is significantly smaller than the pillar diameter, it is unlikely that plasticity in the Al layers is affected by the pillar size.

In addition to dislocation starvation, size effects could also arise from artifacts of the FIB fabrication process. FIB is known to induce amorphization, defect debris, dislocation loops, and ion implantation in some materials between $10-100 \mathrm{~nm}$ below the surface $[33,34,35]$. As these changes only affect a finite damage depth, this may generate a perceived size effect because a larger percentage of a small pillar will be damaged compared to a large pillar. Although this may be a factor in some material systems, Al-SiC nanolaminates have not shown significant FIB damage layers. TEM observations from previous studies on Al-SiC pillars [23] appear to show no increase in dislocation density at the pillar surface and an amorphous layer which is only $\sim 4$ $\mathrm{nm}$ thick. Since the pillar diameters in this study were on the order of micrometers, an amorphous layer of a few nm should have a negligible effect on the overall mechanical response. Another possible mechanism for the strengthening due to the limited size pillars that has not been proposed before and that would be intimately linked to the observed cracking of the $\mathrm{SiC}$ layers is the initial distribution of flaws within each micropillar. This type of size effect has been studied extensively in the ceramics literature using Weibull statistics [36], where the materials fracture strength decreases in larger samples because there is a higher probability for the sample to contain a strength limiting flaw. Although this approach is most often used in tensile or bending tests, the same type of analysis has been successfully applied to compressive failures $[37,38]$. One caveat to using this analysis for compressive loading cases is that cracks could be 
propagated though mode I cracking in the case of wing cracks or mode II cracks due to the resolved shear stress at $45^{\circ}$. As our study is only concerned with determining if the apparent size effect can be attributed to a distribution of flaws, as long as the type of crack propagation is consistent in all pillars used in the analysis, the cracking mode should not affect the dependence on the pillar size.

There are indications which support the idea of a preexisting flaw based failure mechanism in these pillars leading to a size effect. Firstly, small amounts of porosity are observed in these laminates [6,24], often in the troughs of the layer waviness where the uneven surface can cause shadowing during the deposition. Secondly, the largest size effect is observed in the $90^{\circ}$ orientation, where the fracture behavior appears the most brittle (Figure 3(c)), while the effect is greatly reduced in the $45^{\circ}$ oriented pillars, which deform predominantly by plastic shear of the Al layers. To test this hypothesis, 11 additional pillars of both the $1 \times 2 \mu \mathrm{m}$ and $2 \times 4$ $\mu \mathrm{m} 90^{\circ}$ orientation were made using the same procedure outlined above, in order to have an acceptable sample size. Each pillar was strained to failure and the fracture stress was recorded (for consistency across all tests this was taken to be the first instance where the hardening rate becomes negative). The fracture stress values were assigned a probability of survival based on the proportion of pillars which failed at a lower stress. The two parameter Weibull distribution function can relate this probability of survival, $\mathrm{P}_{\mathrm{s}}$, to the fracture stress, $\sigma$, and the sample volume, $\mathrm{V}$, according to the following equation:

$$
P_{S}=\exp \left[-\frac{V}{V_{o}}\left(\frac{\sigma}{\sigma_{o}}\right)^{m}\right]
$$

where $\mathrm{m}$ is the Weibull modulus and the constants $\mathrm{V}_{\mathrm{o}}$ and $\sigma_{\mathrm{o}}$ are the characteristic volume, and the characteristic strength, respectively [32]. Algebraic manipulation of this equation yields the linear form: 


\subsection{Pillar aspect ratio effects}

Finally, by comparing the $2 \times 4 \mu \mathrm{m}$ and $2 \times 6 \mu \mathrm{m}$ pillar stress-strain curves shown in Figure 6, it can be concluded that there is little effect on the mechanical behavior due to altering the aspect ratio. The one notable exception to this is the $0^{\circ}$ orientation. Although the fracture stresses for the two geometries are statistically equivalent in this case as well, the fracture strain is substantially smaller in the 2 x $6 \mu \mathrm{m}$ case. FEA was used to simulate the deformation in both geometries, as shown in Figure 8. The modeling results also displayed a decrease in the strain to failure at larger aspect ratios. This difference stems from the buckling deformation behavior seen in the $0^{\circ}$ orientation. Buckling deformation is a type of plastic instability, the extent of which is determined by the geometry of the initial waviness. This leads to the buckled region having the same height in both modeled geometries (Figure 8). Therefore, in the case of the high aspect ratio pillars, the wavelength is a smaller proportion of the overall pillar height, leading to a smaller apparent strain before failure. 


\section{Conclusions}

In this work, the effects of orientation, pillar geometry, and layer morphology were investigated though a combination of experimental observations and FEA. In light of the results obtained, the following conclusions can be made:

- The $0^{\circ}$ orientation shows the highest strength because the reinforcing SiC layers are aligned with the loading axis while the $45^{\circ}$ orientation shows the lowest strength because it accommodates shear deformation more easily.

- The deformation behavior in the $0^{\circ}$ and $45^{\circ}$ orientations is highly dependent on the waviness of the layer structure because deformation is limited by bending and buckling of the layers.

- The maximum strength of the $90^{\circ}$ orientation is limited by cracking of the $\mathrm{SiC}$ layers.

- A size effect is observed in all orientations, but more predominantly in the $0^{\circ}$ and $90^{\circ}$ orientations. Using a Weibull statistics approach, it was shown that the difference in strength can be attributed to the lower probability of the smaller pillars containing a strength limiting flaw.

- An aspect ratio effect is observed in only the $0^{\circ}$ orientation where the failure strain decreases at higher aspect ratios. This is due to the majority of the deformation being accommodated by a buckling type of plastic instability, the size of which is not related to the pillar height, but to the geometry of the existing layer undulation.

\section{Acknowledgements}


The authors would like to acknowledge the National Science Foundation Materials World Network (Contract DMR-1209928, Dr. Lynnette Madsen, Program Manager) and the Spanish Ministry of Economy (PCIN-2013-029) for financial support of this research. We are also grateful to the LeRoy Erying Center for Solid State Science for the use of their microscopy facilities. JMM-A is also indebted to the Fulbright commission and the Spanish Ministry of Education for his visiting scholarship (PRX14/00439). Sample fabrication was performed at the Center for Integrated Nanotechnologies, an Office of Science User Facility operated for the U.S. Department of Energy (DOE) Office of Science by Los Alamos National Laboratory (Contract DE-AC52-06NA25396) and Sandia National Laboratories (Contract DE-AC04-94AL85000). 


\section{References}

[1] M. Shinn, L. Hultman, and S. Barnett, Growth, structure, and microhardness of epitaxial TiN/NbN superlattices, J. of Mater. Res. 7 (1992) 901-911.

[2] U. Wiklund, P. Hedenqvist, and S. Hogmark, Multilayer cracking resistance in bending, Surf. and Coat. Technol. 97 (1997) 773-778.

[3] N. Chawla, D. Singh, Y.-L. Shen, G. Tang, and K. Chawla, Indentation mechanics and fracture behavior of metal/ceramic nanolaminate composites, J. of Mater. Sci. 43 (2008) 43834390.

[4] D. Singh and N. Chawla, Scratch resistance of Al/SiC metal/ceramic nanolaminates, J. of Mater. Res. 27 (2012) 278-283.

[5] N. A. Mara, D. Bhattacharyya, P. Dickerson, R. G. Hoagland, and A. Misra, Deformability of ultrahigh strength 5nm $\mathrm{Cu}-\mathrm{Nb}$ nanolayered composites, Appl. Phys. Lett. 92 (2008) 1-3.

[6] D. Singh, N. Chawla, G. Tang, and Y.-L. Shen, Micropillar compression of Al/SiC nanolaminates," Acta Materialia, 58 (2010) 6628-6636.

[7] W. Li, B. Kabius, and O. Auciello, Science and technology of biocompatible thin films for implantable biomedical devices, in Eng. in Med. and Biol. Soc. Annual International Conference of the IEEE (2010) 6237-6242.

[8] G. S. Hickey, S.-S. Lih, and T. W. Barbee, Jr., Development of nanolaminate thin-shell mirrors, Proc. SPIE 4849 (2002) 63-76.

[9] R. O. Ritchie, The conflicts between strength and toughness, Nat. mater. 10 (2011) 817822. 
[10] M. F. Ashby, H. Shercliff, and D. Cebon, Materials: engineering, science, processing and design. Butterworth-Heinemann, 2013.

[11] K. Hwu and B. Derby, Fracture of metal/ceramic laminates II. crack growth resistance and toughness, Acta Materialia, vol. 47 (1999) 545 - 563.

[12] S. Roy, B. Butz, and A. Wanner, Damage evolution and domain-level anisotropy in metal/ceramic composites exhibiting lamellar microstructures, Acta Materialia, 58 (2010) 2300 2312.

[13] R. R. Adharapurapu, K. S. Vecchio, F. Jiang, and A. Rohatgi, Effects of ductile laminate thickness, volume fraction, and orientation on fatigue-crack propagation in $\mathrm{Ti}^{-} \mathrm{Al}_{3} \mathrm{Ti}$ metalintermetallic laminate composites, Metall. and Mater. Trans. A 36 (2005) 1595-1608.

[14] M. D. Uchic and D. M. Dimiduk, A methodology to investigate size scale effects in crystalline plasticity using uniaxial compression testing, Mater. Sci. and Eng. A 400 (2005) 268278.

[15] J. R. Greer, W. C. Oliver, and W. D. Nix, Size dependence of mechanical properties of gold at the micron scale in the absence of strain gradients, Acta Materialia 53 (2005) 1821-1830. [16] J. R. Greer, C. R. Weinberger, and W. Cai, Comparing the strength of f.c.c. and b.c.c. sub-micrometer pillars: Compression experiments and dislocation dynamics simulations, Mater. Sci. and Eng. A 493 (2008) 21-25.

[17] E.-Y. Guo, H.-X. Xie, S. S. Singh, A. Kirubanandham, T. Jing, and N. Chawla, Mechanical characterization of microconstituents in a cast duplex stainless steel by micropillar compression, Mater. Sci. and Eng. A 598 (2014) 98-105.

[18] C. A. Volkert and E. T. Lilleodden, Size effects in the deformation of sub-micron Au columns, Phil. Mag. 86 (2006) pp. 5567-5579. 
[19] J. Zhang, X. Liang, P. Zhang, K. Wu, G. Liu, and J. Sun, Emergence of external size effects in the bulk-scale polycrystal to small-scale single-crystal transition: A maximum in the strength and strain-rate sensitivity of multicrystalline Cu micropillars, Acta Materialia 66 (2014) 302-316.

[20] C. Mayer, L. Yang, S. Singh, H. Xie, Y.-L. Shen, J. Llorca, J. Molina-Aldareguia, and N. Chawla, Orientation dependence of indentation behavior in Al-SiC nanolaminate composites, Mater. Lett. 168 (2016) 129-133.

[21] S. Pathak and S. R. Kalidindi, Spherical nanoindentation stress-strain curves, Mater. Sci. and Eng $R 91$ (2015) 1-36.

[22] C. Chen, Y. Pei, and J. D. Hosson, Effects of size on the mechanical response of metallic glasses investigated through in situ tem bending and compression experiments, Acta Materialia 58 (2010) 189-200.

[23] S. Lotfian, M. Rodriguez, K. Yazzie, N. Chawla, J. Llorca, and J. Molina-Aldareguia, High temperature micropillar compression of Al/SiC nanolaminates, Acta Materialia 61 (2013) 4439-4451.

[24] C. Mayer, N. Li, N. Mara, and N. Chawla, Micromechanical and in situ shear testing of Al-SiC nanolaminate composites in a transmission electron microscope (tem)," Mater. Sci. and Eng. A 621 (2015) 229-235.

[25] L. Yang, C. Mayer, N. Chawla, J. LLorca, and J. M. Molina-Aldareguía, Deformation mechanisms of ultra-thin $\mathrm{Al}$ layers in $\mathrm{Al} / \mathrm{SiC}$ nanolaminates as a function of thickness and temperature, Phil. Mag. (2016) in revision. 
[26] T. Nizolek, N. Mara, R. McCabe, J. Avallone, I. Beyerlein, and T. Pollock, Kink band formation in high-strength bulk metallic nanolaminates, Microsc. and Microanal. 21 (2015) $1157-1158$.

[27] G. S. Bales and A. Zangwill, Macroscopic model for columnar growth of amorphous films by sputter deposition, J. of Vac. Sci. \& Tech. A 9 (1991) 145-149.

[28] B. Lauterbach and D. Gross, Crack growth in brittle solids under compression, Mech. of Mater. 29 (1998) 81-92.

[29] J. R. Greer and J. T. D. Hosson, Plasticity in small-sized metallic systems: Intrinsic versus extrinsic size effect, Prog. in Mater. Sci. 56 (2011) 654-724.

[30] M. Tschopp and D. McDowell, Grain boundary dislocation sources in nanocrystalline copper, Scripta Materialia 58 (2008) 299-302.

[31] A. Misra, J. Hirth, and R. Hoagland, Length-scale-dependent deformation mechanisms in incoherent metallic multilayered composites, Acta Materialia 53 (2005) 4817-4824.

[32] P. Sun, J. Chu, T. Lin, Y. Shen, and N. Chawla, Characterization of nanoindentation damage in metal/ceramic multilayered films by transmission electron microscopy (tem), Mater. Sci. and Eng. A 527 (2010) 2985-2992.

[33] J. A. El-Awady, C. Woodward, D. M. Dimiduk, and N. M. Ghoniem, Effects of focused ion beam induced damage on the plasticity of micropillars, Phys. Rev. B 80 (2009) 104104:1-5.

[34] S. Shim, H. Bei, M. Miller, G. Pharr, and E. George, Effects of focused ion beam milling on the compressive behavior of directionally solidified micropillars and the nanoindentation response of an electropolished surface, Acta Materialia, 57 (2009) 503-510. 
[35] H. Bei, S. Shim, M. K. Miller, G. M. Pharr, and E. P. George, Effects of focused ion beam milling on the nanomechanical behavior of a molybdenum-alloy single crystal, Appl. Phys. Lett. 91 (2007) 111915:1-3.

[36] R. Danzer, P. Supancic, J. Pascual, and T. Lube, Fracture statistics of ceramics: Weibull statistics and deviations from weibull statistics, Eng. Fract. Mech. 74 (2007) 2919-2932.

[37] J. Huang, S. Xu, H. Yi, and S. Hu, Size effect on the compression breakage strengths of glass particles, Powder Tech. 268 (2014) 86-94.

[38] T. Wong, R. H. Wong, K. Chau, and C. Tang, Microcrack statistics, weibull distribution and micromechanical modeling of compressive failure in rock, Mech of Mater. 38 (2006) 664681. 


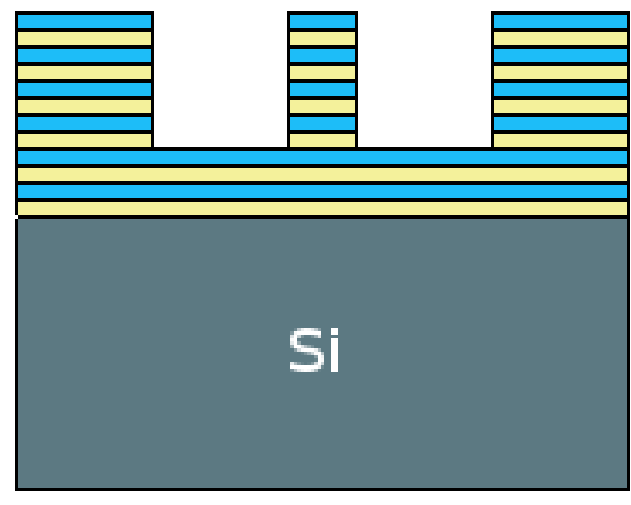

90 Degree

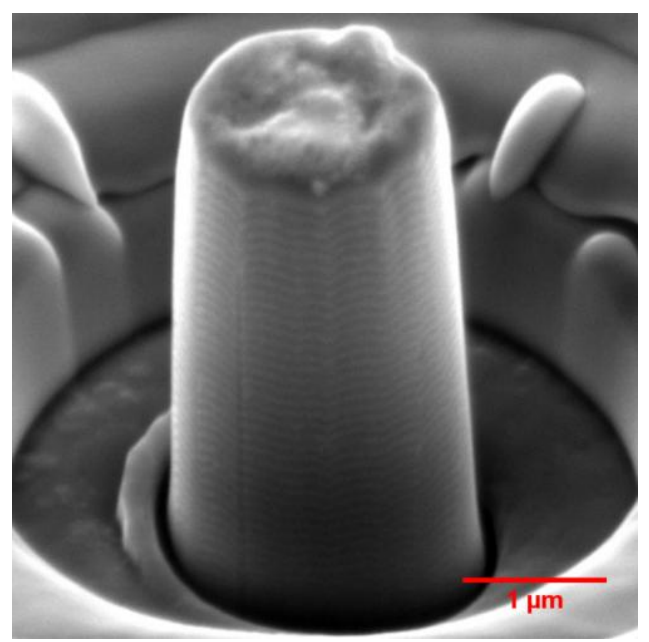

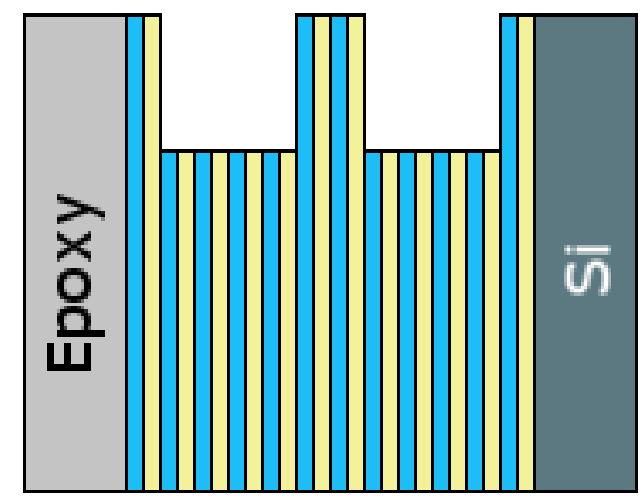

0 Degree

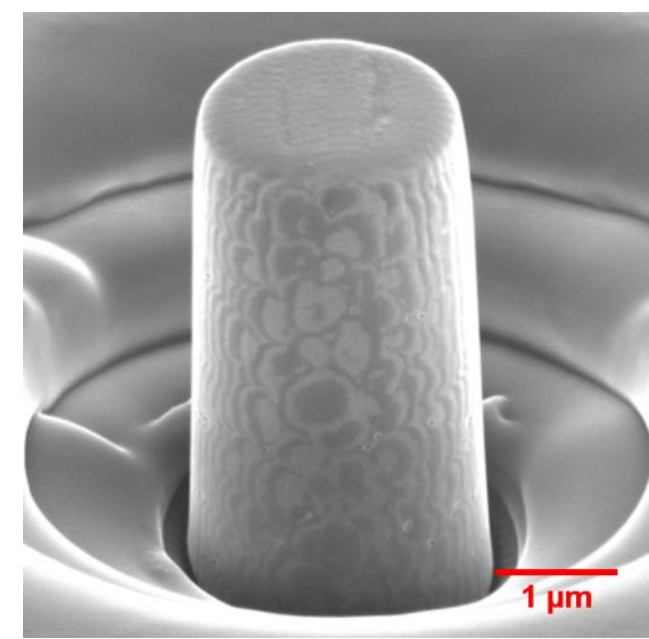

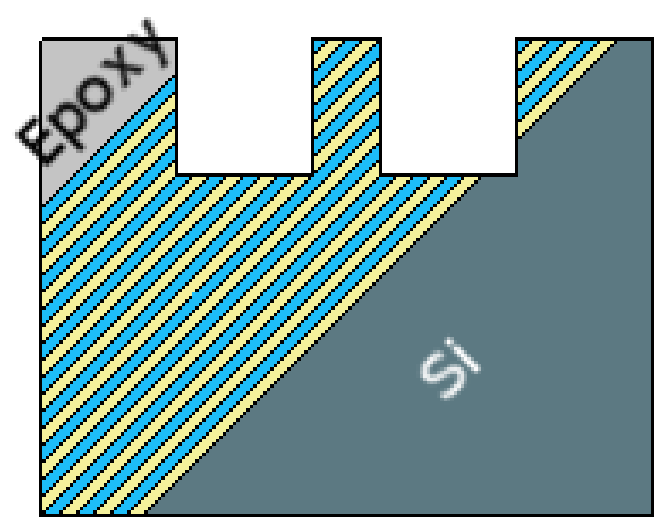

45 Degree

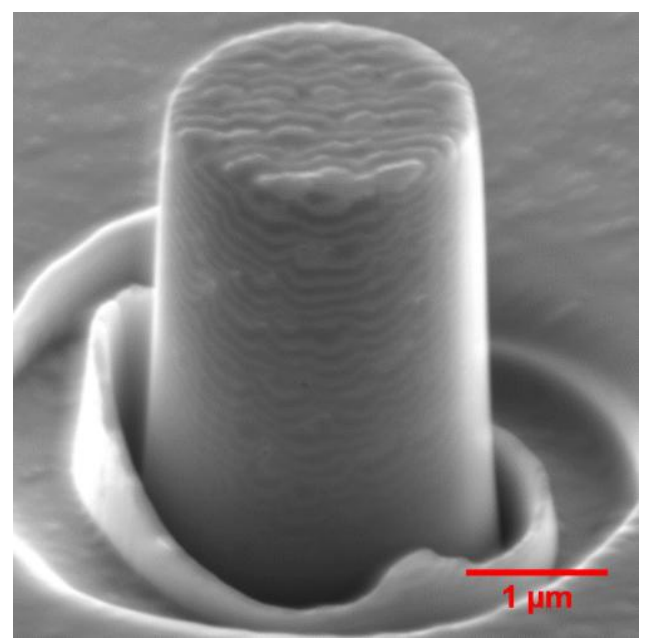

Figure 1: Schematic of the pillar orientations tested and SEM images of $2 \times 4 \mu \mathrm{m}$ pillars prior to testing. 

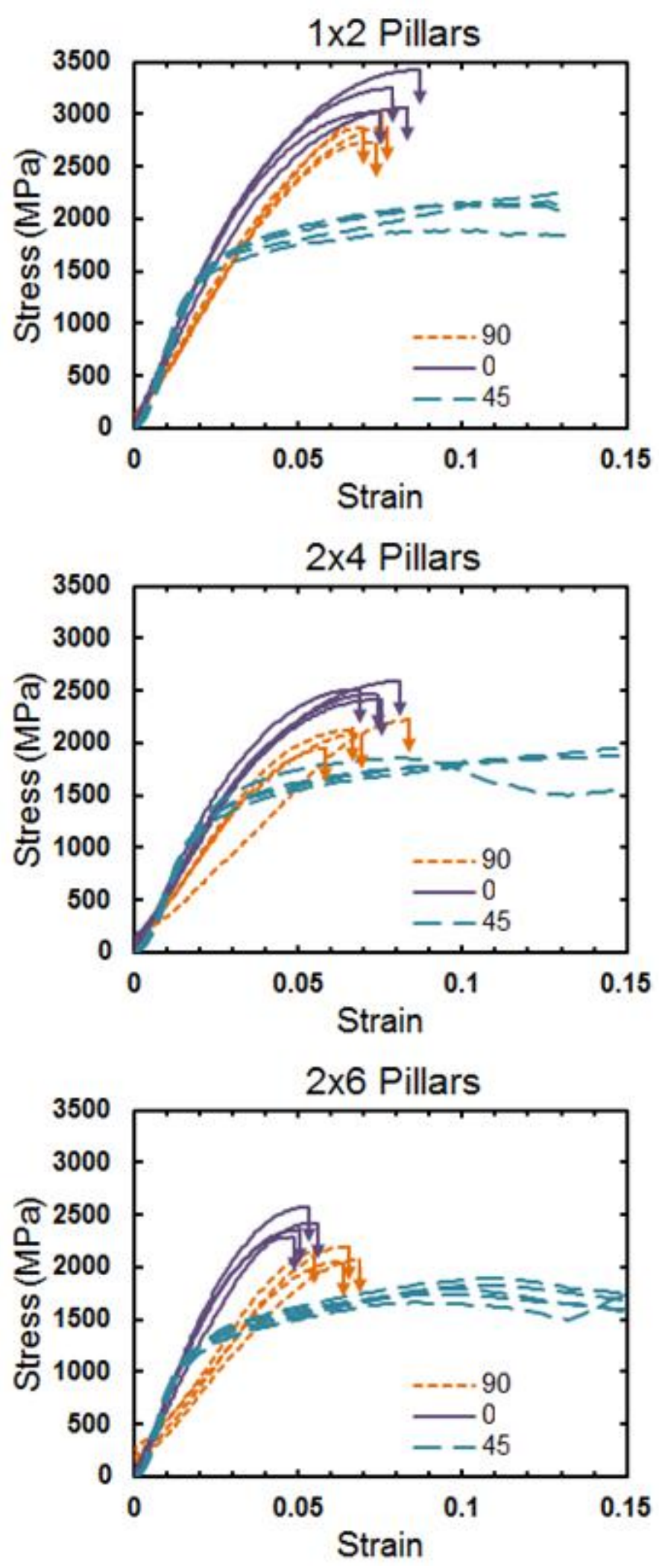

Figure 2: Experimental engineering stress strain curves showing the effect of layer orientation on mechanical response for different geometries. 

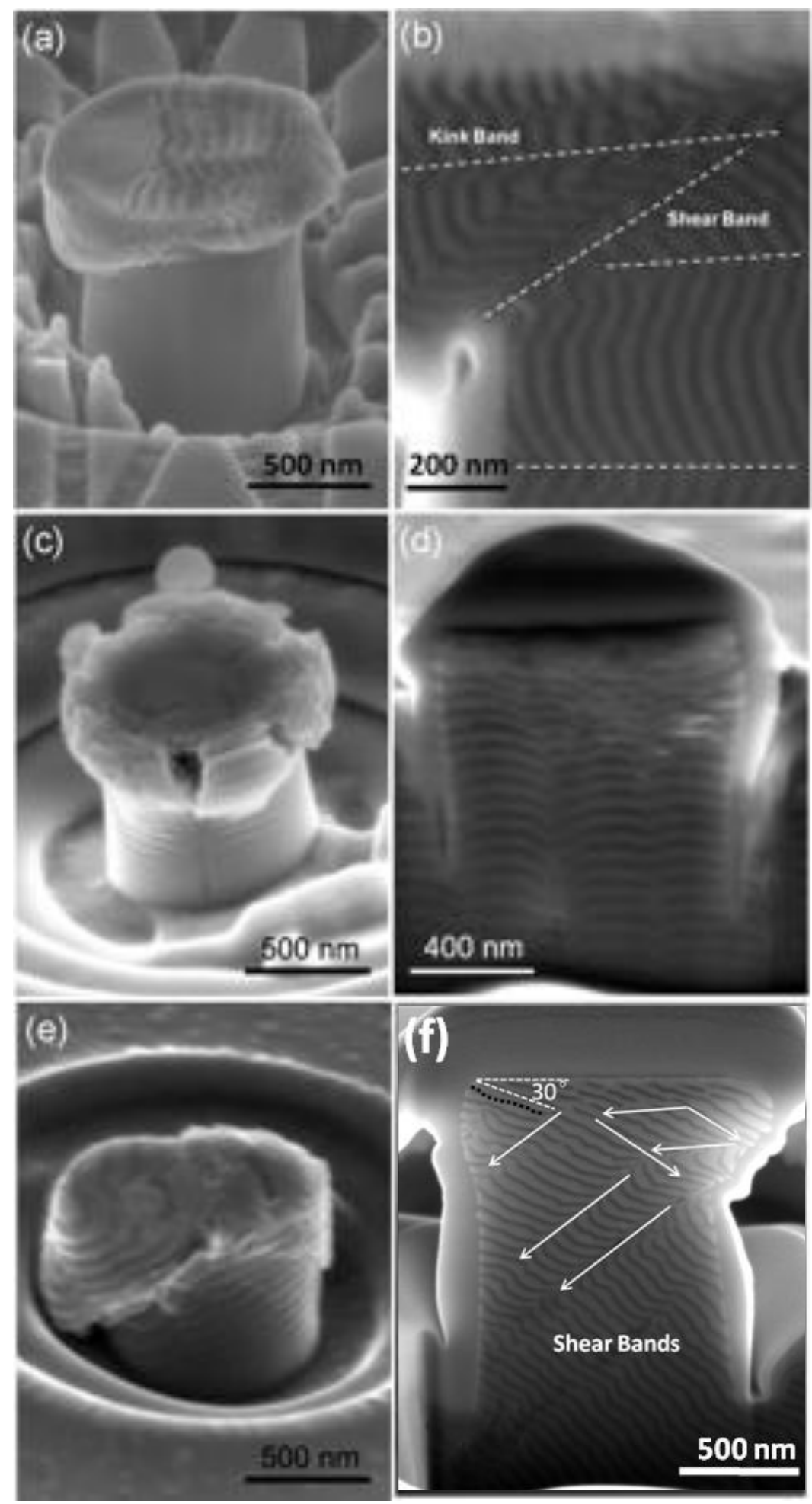

Figure 3: SEM images of $1 \mathrm{x} 2 \mu \mathrm{m}$ pillars and cross sections following compression for $0^{\circ}$ (a and b), $90^{\circ}$ (c and d), and $45^{\circ}$ (e and f) orientations. The deformation behavior shows large differences with the $0^{\circ}$ and $45^{\circ}$ orientations being strongly influenced by the buckling of the layers. The $90^{\circ}$ orientation shows extrusion of the Al layers. 


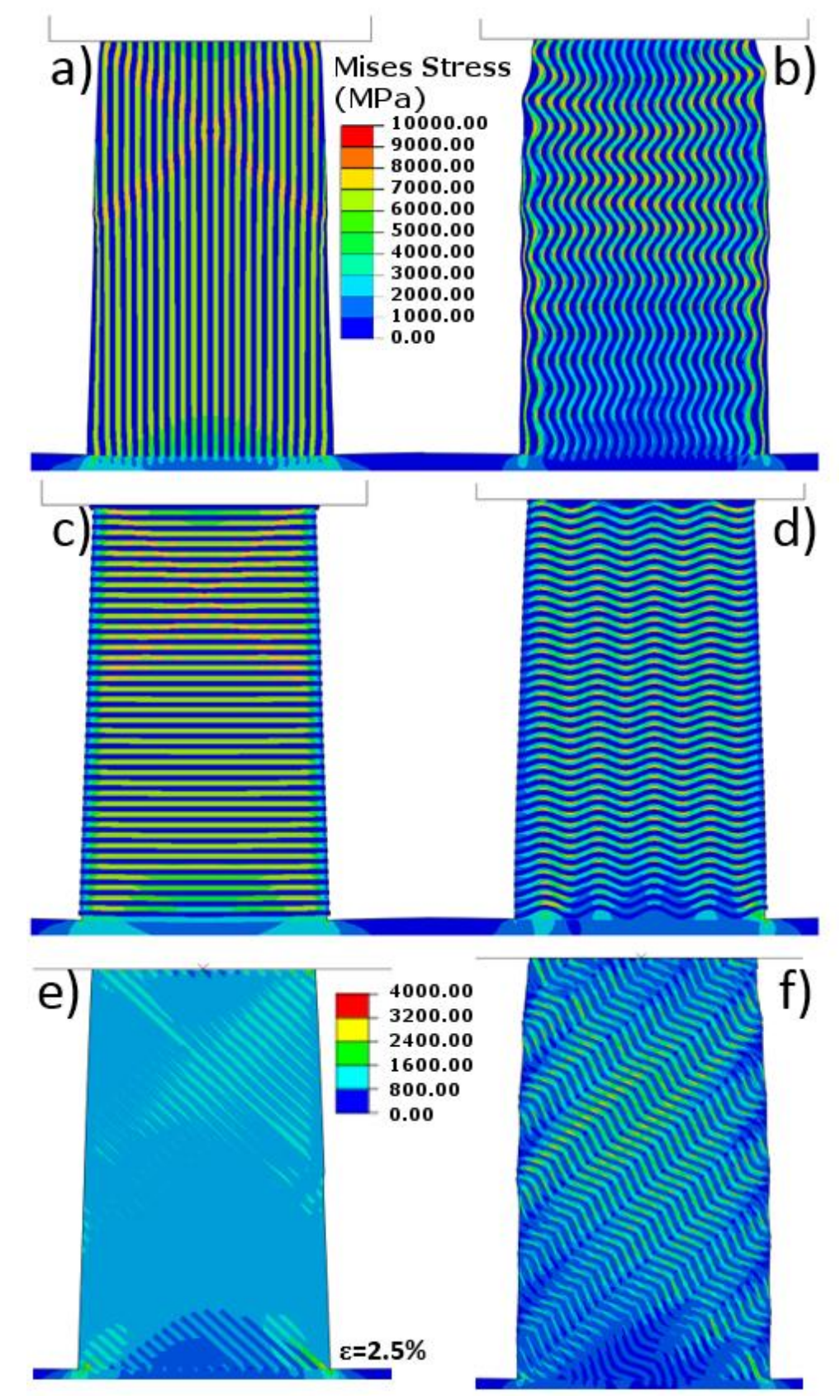

Figure 4: FEA stress contours at $6 \%$ strain showing the effect of $0 \mathrm{~nm}$ (a, c, and e) and $45 \mathrm{~nm}$ (b, $\mathrm{d}$, and $\mathrm{f}$ ) amplitude waviness on the deformation behavior for all three orientations. Due to lack of convergence e) is only able to be modeled to $2.5 \%$ strain (also note the difference in contour levels). 

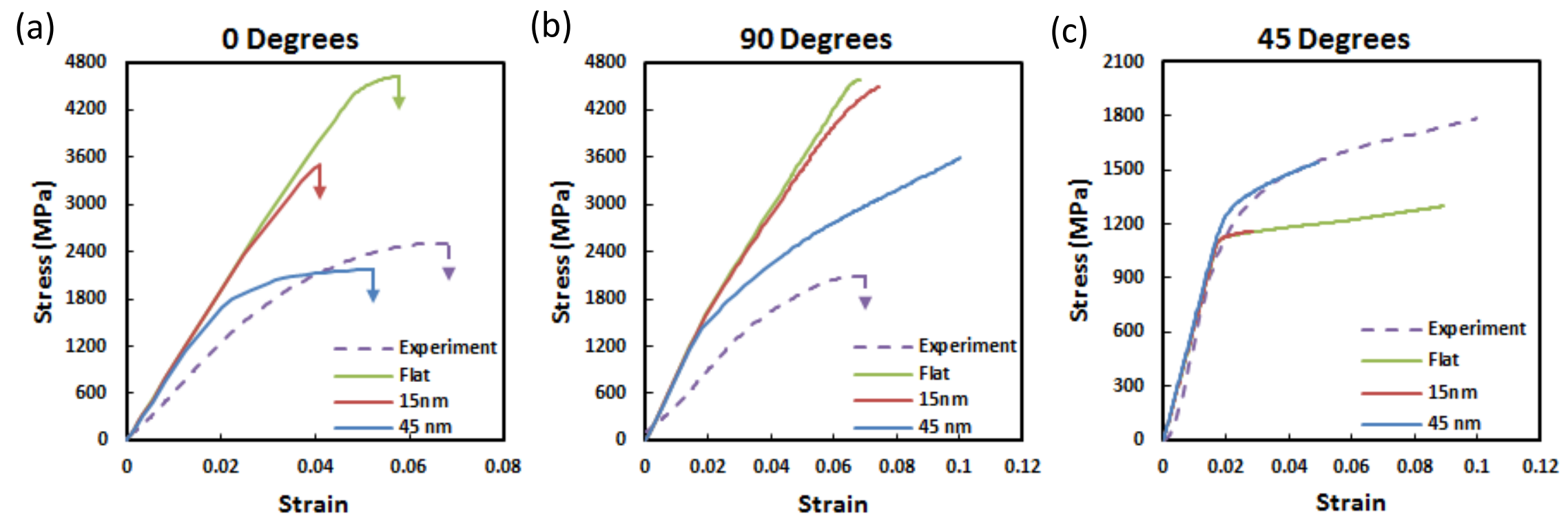

Figure 5: Comparison of engineering stress strain curves for a representative $2 \times 4 \mu \mathrm{m}$ pillar to FEA simulations (also $2 \times 4 \mu \mathrm{m})$ having waviness amplitudes of 0,15 and $45 \mathrm{~nm}$ in each orientation. 

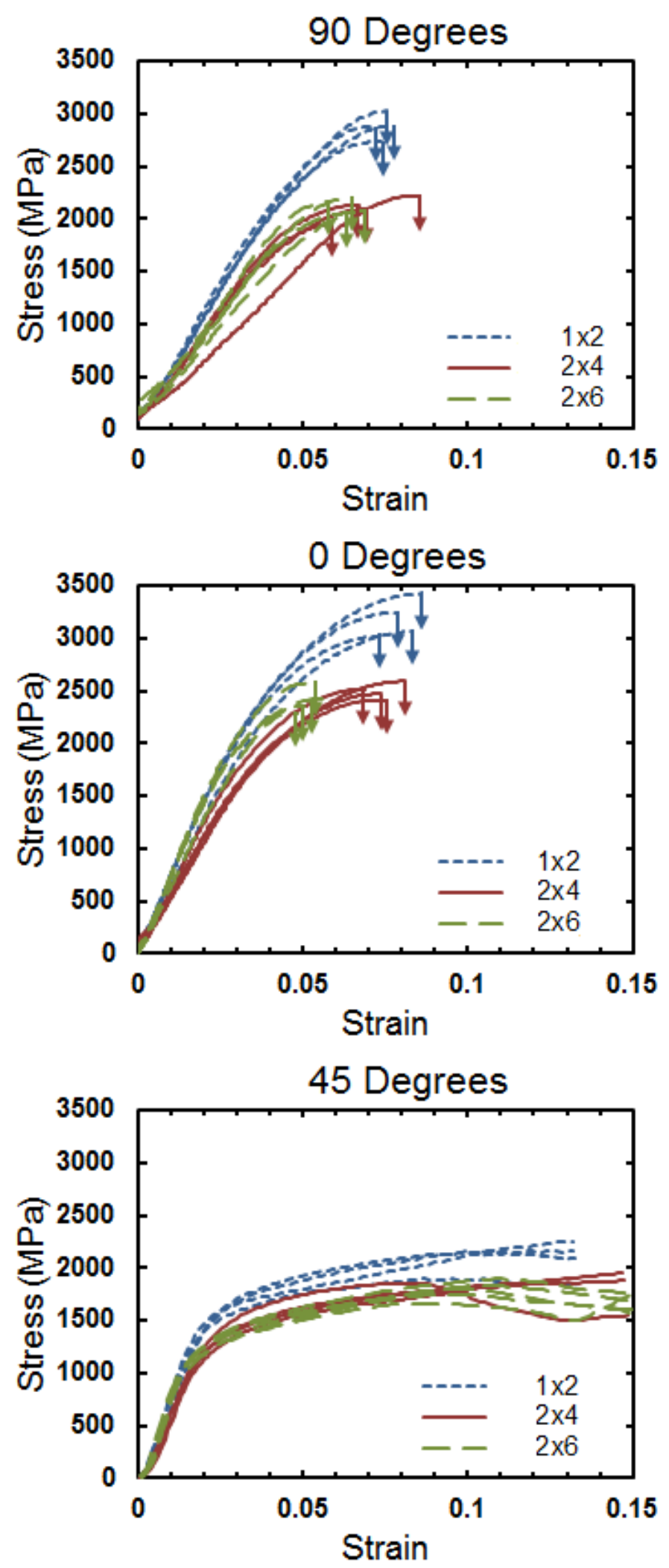

Figure 6: Experimental engineering stress-strain curves showing the effect of pillar size and aspect ratio on mechanical behavior. 
Figure 7: Weibull plot of the fracture stresses of $1 \times 2$ and $2 \times 4 \mu \mathrm{m}$ pillars (in the $90^{\circ}$ orientation). The fact that both sets of data fall on a single linear fit line indicates that the increase in mean strength of the $1 \times 2 \mu \mathrm{m}$ pillars can be attributed to lower probability of a strength limiting flaw and is not due to FIB damage or a true material size effect. 

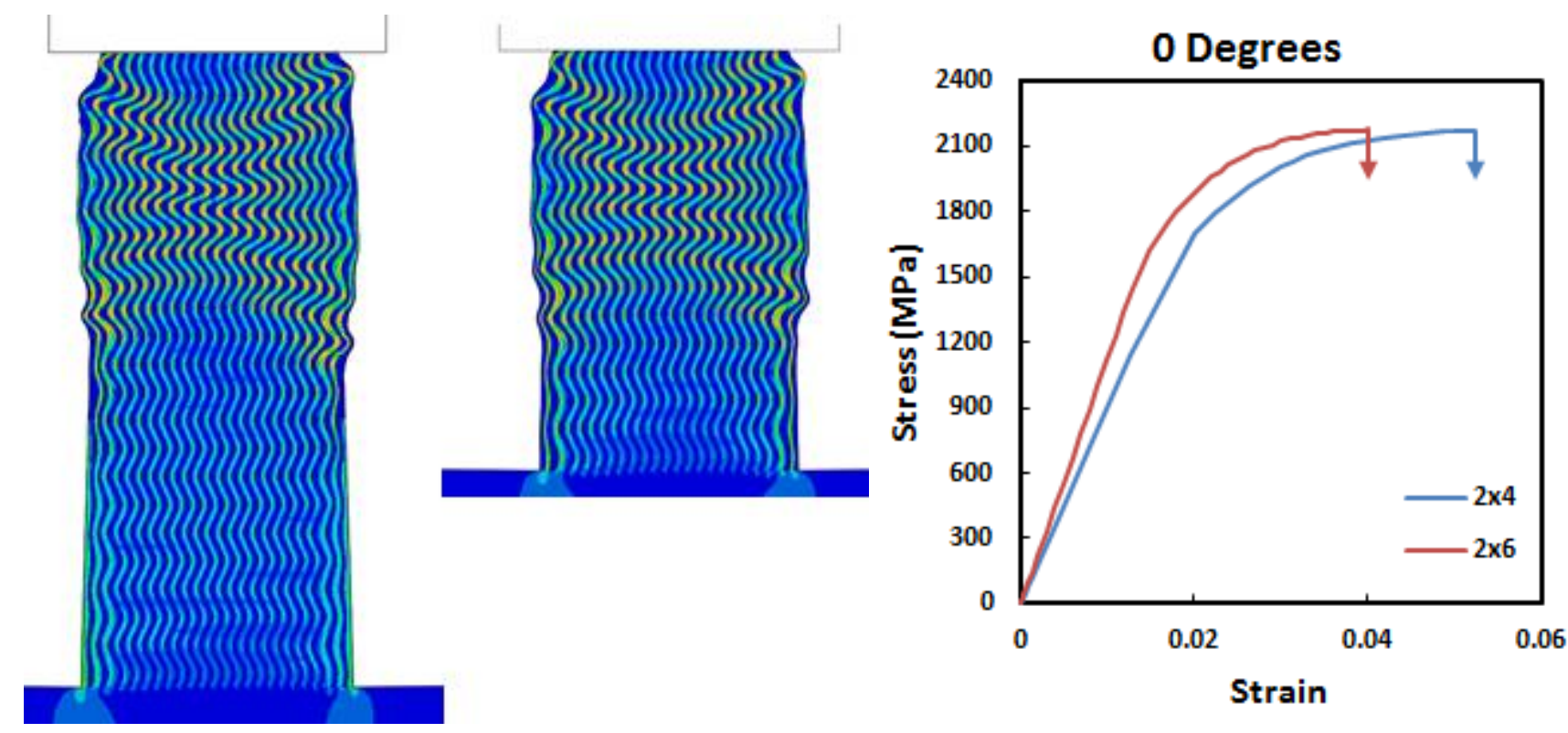

Figure 8: Simulated deformation behavior of $2 \times 6$ and $2 \times 4 \mu \mathrm{m}$ pillars (same contour scale as Figure 4) showing that the height of the instability is independent of the overall pillar height. This leads to a decreased apparent strain to failure, as shown in the engineering stress-strain curves. 
Anisotropy, Size, and Aspect Ratio Effects on Micropillar Compression of Al-SiC Nanolaminate Composites

C.R. Mayer, L.W. Yang, S.S. Singh, J. LLorca, J.M. Molina-Aldareguia, Y.L. Shen, N. Chawla

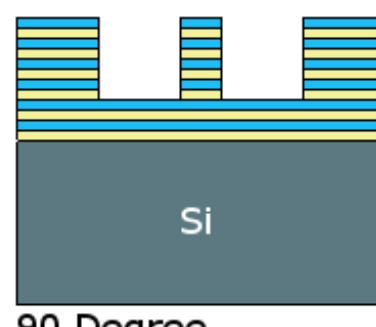

90 Degree
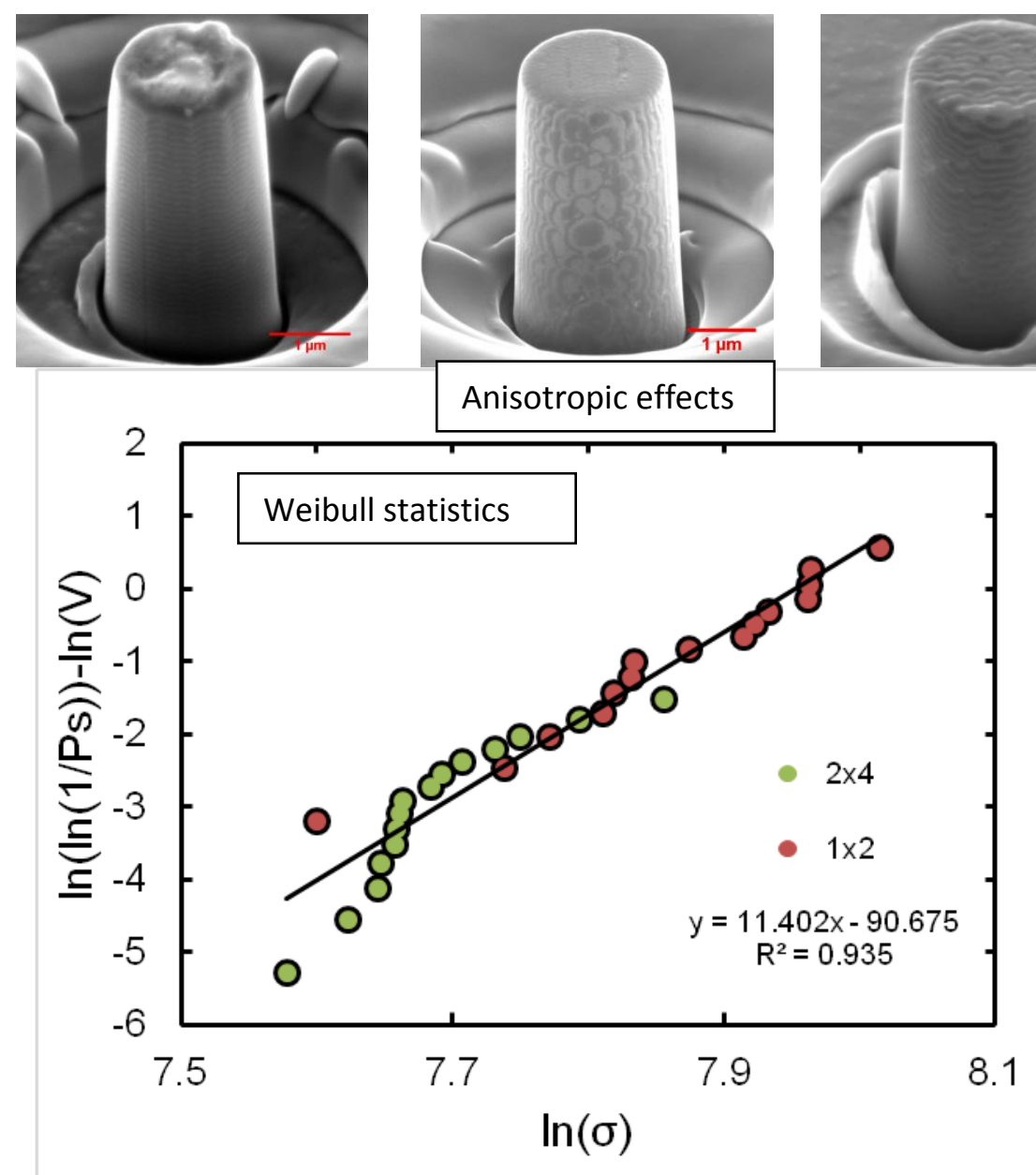
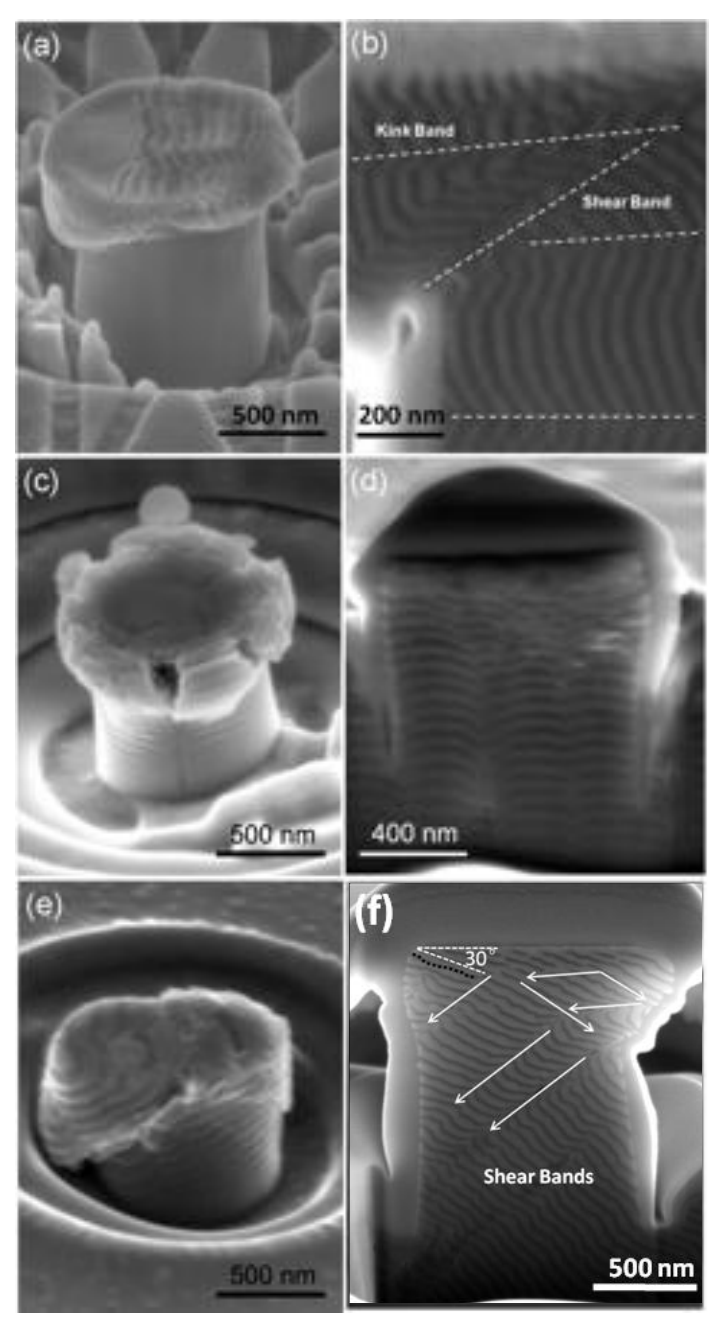

Damage mechanisms 